\title{
ISCHAEMIC SENSORY LOSS IN PATIENTS WITH SPINAL AND CEREBRAL LESIONS
}

\author{
BY \\ R. W. GILLIATT \\ From The National Hospital, Queen Square, London
}

In an investigation which has already been reported (Gilliatt and Wilson, 1954), sensation in the fingers was studied during periods of ischaemia of the arm, the ischaemia being produced artificially by inflating pneumatic cuffs to above arterial pressure round the limb. In a group of normal subjects examined after the inflation of a pneumatic tourniquet above the elbow, the mean duration of ischaemia required to produce subjective numbness of the finger-tips was 14 minutes, but when patients with peripheral nerve lesions were tested in this way, it was found that sensation failed with abnormal rapidity in areas of skin supplied by a damaged nerve, so that an area in which sensation was initially only mildly disturbed might, within five or 10 minutes, become sharply demarcated from the rest of the hand.

The abnormally rapid failure of sensation during ischaemia in an area of initial sensory impairment did not appear to be due solely to the local effect of asphyxia on damaged fibres, as it occurred in patients with dorsal root lesions and with nerve lesions proximal to the site of the pneumatic cuff. As an explanation it was suggested that while some of the sensory fibres in an ischaemic nerve would be blocked by the ischaemia, a proportion would continue to conduct impulses under the pneumatic cuff; of these, however, a further proportion would be blocked by any lesion at a higher level, so that the total effect on sensation would be an additive one.

This suggested that a sensory deficit at any level in the nervous system might be exaggerated by the addition of a partial peripheral nerve block, the peripheral block by itself being insufficient to produce sensory loss. The present investigation of patients with spinal and cerebral lesions was undertaken to explore this possibility.

\section{METHODS}

These differed in certain details from those described in the previous paper. Whereas previously a rubber cuff 6 in. by 15 in. was used, the present experiments were carried out with a standard sphygmomanometer cuff 5 in. by $9 \mathrm{in}$. In each test the sphygmomanometer armlet was firmly bandaged round the arm above the elbow and then inflated rapidly to 200 to $220 \mathrm{~mm}$. $\mathrm{Hg}$. With the smaller rubber bag the inflation was more rapid than in the earlier experiments, and it was not considered necessary to raise the limb beforehand, as had been done previously to avoid venous congestion. After the inflation of the pneumatic tourniquet the arm was rested comfortably with the palm uppermost, and the finger-pads were firmly rubbed or stroked at halfor one-minute intervals until the patient noticed a change in the sensation aroused.

As in the previous work the onset of subjective numbness of the skin of the fingers was easily recognized when it occurred, touches giving rise to an abnormal sensation variously described as "numb", "dead", or "wooden", as if the fingers had "gone to sleep". Patients were rarely in doubt as to the onset of subjective numbness, but when difficulty was experienced it was usually helpful to ask the patient to feel the affected fingers with the other hand. Subjective numbness sometimes included a rough, prickly component, as described by Weddell and Sinclair (1947), but this was variable, and a few patients seemed to develop complete anaesthesia in the finger-tips without noticing in the intervening stages anything suggesting a rough, prickly, tingling, or electric sensation in response to touch.

The earliest time during ischaemia at which subjective numbness was definitely present in the tips of all the digits has been called the "time to numbness". On a few occasions the "time to numbness" was recorded separately for different fingers, but otherwise the term indicates involvement of the tips of all the digits within the time stated.

In three of the patients subjective numbness was already present in the fingers before ischaemia, and these three were therefore asked to say when they considered that the sensation aroused by a firm touch was definitely less than before the test. This was a more difficult subjective change to assess than the actual onset of numbness, but the patients who were examined in this way did in fact give consistent answers in repeated tests.

The methods used to investigate changes in the tactile threshold were similar to those employed previously, 
FIG. 1.-Effects of ischaemia of the arm in three patients with disseminated sclerosis. In each case five tests per minute with nylon hair on finger tip. Ischaemia at arrow.

1
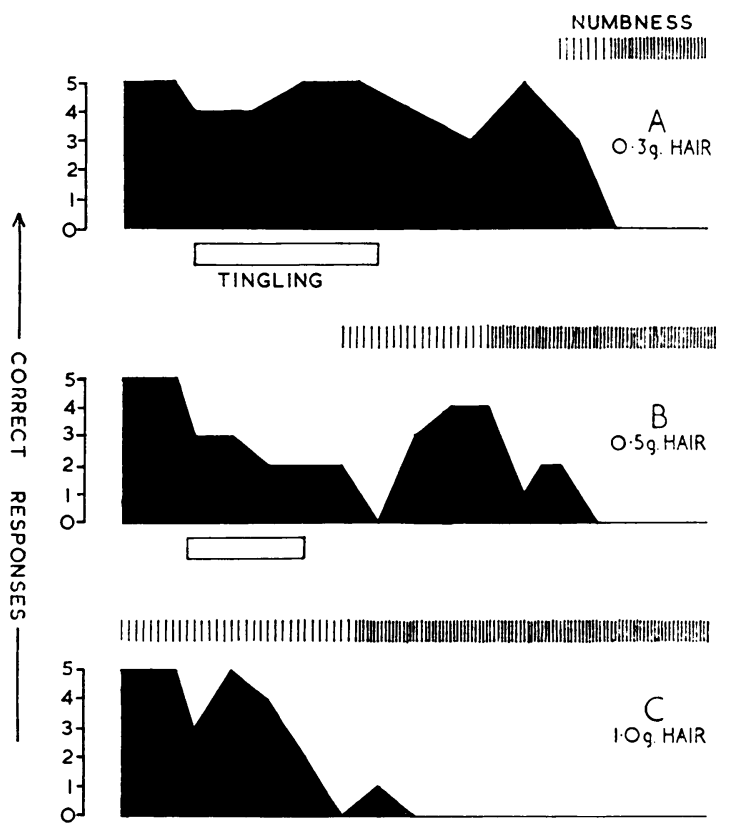

0

5

10

15

and short lengths of standard nylon suture material, suitably mounted and calibrated, were again used to provide graded tactile stimuli. In some patients an area of skin on the finger-pad was ringed and used for testing, but in those with horny skin the dorsum of the terminal phalanx was preferred. Runs of five stimuli were used each minute, the stimuli being spaced irregularly to prevent guessing. The patient's score was then recorded graphically as the number of stimuli perceived each minute. All tests in the present series were carried out at room temperature.

\section{RESULTS}

\section{Patients with Spinal Cord Lesions}

Five patients with lesions of the spinal cord in the cervical region were examined. In two patients with cervical cord tumours, the level of the lesion was not in doubt and was confirmed at operation in each case. Three patients suffered from disseminated sclerosis, and in them it is unlikely that the disease was restricted to the cervical region of the spinal cord, although in each case the presence of symmetrical sensory symptoms in the hands in associa- tion with spastic paraplegia suggested that this region was severely involved (cf. McAlpine, 1954).

When the circulation to the arm was arrested by a pneumatic tourniquet above the elbow, the " time to numbness" was in every case less than the normal figure of 14 minutes, found previously in 50 control subjects (Gilliatt and Wilson, 1954). In some experiments the "time to numbness" was reduced to less than half the normal figure, and in only two cases did it exceed 10 minutes.

When the effects of ischaemia are compared closely with the results of formal sensory testing it is clear that in general the "time to numbness" was shortest in those with the most severe sensory defects. This is well illustrated by the three patients with disseminated sclerosis (Fig. 1).

Case 1.-Mrs. H. N., aged 52 (N.H. No. 19317), was admitted to the National Hospital under the care of Dr. Denis Brinton on December 13, 1952, with a history of increasing stiffness and weakness of the legs for five years, with occasional loss of sphincter control. Intermittent "pins and needles" in the fingers of both hands were first noticed in 1950, and more recently the fingers had felt numb and had tingled when they were touched.

Examination on admission in December, 1952, revealed pallor of both optic discs but no other abnormality in the cranial nerves. Tone and power in the arms were normal, but the tendon reflexes were abnormally brisk and tactile sensation was impaired in both hands. Severe spastic weakness of both legs was present with extensor plantar responses. Superficial sensation was present in the legs, but vibration sensation was absent below the costal margin, and passive movements of the toes were not perceived.

Sensation in the hands was examined in detail before a tourniquet test on March 1 . There had been some improvement since admission, and the fingers no longer felt numb although they still tingled faintly when they were touched. Pain and temperature were perceived normally and light touches with a $0.3 \mathrm{~g}$. nylon hair were felt without difficulty on the finger-tips. There were errors in discrimination of compass points on the finger-pads at $0.5 \mathrm{~cm}$. separation but not at $0.7 \mathrm{~cm}$. Vibration and joint sensation were normal.

The effects of ischaemia of the right arm are shown in Fig. 1A. A sphygmomanometer cuff was inflated to $210 \mathrm{~mm}$. $\mathrm{Hg}$ round the arm above the elbow, and within one minute the patient noticed ischaemic tingling in the fingers which persisted for five minutes. During the first 10 minutes of ischaemia the patient missed occasional touches with the $0.3 \mathrm{~g}$. hair but the skin of the fingers did not feel abnormal when it was rubbed until 11 minutes, when the finger-tips were described as " a bit changed and numb". After $12 \frac{1}{2}$ minutes the fingers had "definitely gone to sleep", and at this time touches with the nylon hair could no longer be perceived.

Comment.-Although in the past this patient had suffered from tingling and numbness of the fingers, 
these symptoms had improved, and at the time of testing the hands were normal save for the presence of faint paraesthesiae in the finger-tips and mildly defective two-point discrimination. When the circulation to the arm was arrested by a pneumatic tourniquet above the elbow, subjective numbness of the fingers did not appear until 11 minutes after the onset of ischaemia. Light touches with a $0 \cdot 3 \mathrm{~g}$. hair were still appreciated at 10 minutes, but were missed after $12 \frac{1}{2}$ minutes.

Case 2.-Mrs. A. B., aged 27 (N.H. No. 26919), was admitted to the National Hospital under the care of Dr. Purdon Martin on March 6, 1953, with a history of fluctuating weakness of the legs for three years and of clumsiness of the hands for six months. Examination on admission revealed pathological pallor of both optic discs with a small central scotoma on the right. In the arms tone and power were normal, but the tendon reflexes were abnormally brisk. A severe spastic paraplegia was present with an ill-defined upper level of sensory loss in the lower dorsal region.

On admission the patient complained of numbness of the fingers of both hands with tingling when they were touched, but by the time that an ischaemic test was carried out on March 17 sensation had improved and subjectively the skin felt normal. Pain and temperature were perceived normally, but light touches with a $0.3 \mathrm{~g}$. nylon hair were often missed and there were many errors in two-point discrimination at $0.5 \mathrm{~cm}$. on the fingers. Vibration and joint sensation in the fingers were normal.

A sphygmomanometer cuff was inflated to $210 \mathrm{~mm}$. $\mathrm{Hg}$ round the right arm above the elbow (Fig. 1B), and resulted in faint ischaemic paraesthesiae which lasted for three minutes. After five minutes the finger-tips felt numb when they were touched and by nine minutes "much more dead". A $0.5 \mathrm{~g}$. nylon hair was used to test sensation on the finger-pads as the patient perceived this stimulus without difficulty before ischaemia. During the ischaemic tingling, however, touches with the hair were often missed, and after the paraesthesiae ceased the patient's appreciation of the stimulus improved only slightly before failing altogether after 12 minutes of ischaemia.

Comment.-In this patient the tactile threshold was initially slightly raised in the finger-tips, and ischaemia of the arm resulted in more rapid deterioration in sensation than in Case 1, subjective numbness of the fingers occurring after five minutes with complete anaesthesia to a $0.5 \mathrm{~g}$. hair after 12 minutes.

Case 3.-Miss E. W., aged 41 (N.H. No. 10332), was admitted to the National Hospital under the care of Dr. Carmichael on February 12, 1954, with a history of weakness of the legs since 1941, at which time an episode of impaired vision in the left eye had occurred. Examination at the National Hospital in 1945 had shown pallor of the left optic disc, absent abdominal reflexes, and a spastic paraparesis. The arms remained normal until 1951, but thereafter the patient noticed increasing stiffness and clumsiness with numbness of the skin of the hands.

Examination on admission in February, 1954, revealed a severely disabled patient with bilateral optic atrophy, nystagmus, and spastic weakness of all four limbs. All forms of sensation were impaired in the lower limbs and on the trunk below the level of D2, but appreciation of pain and temperature was preserved in the upper limbs. There was, however, subjective numbness of the skin of both hands with a raised tactile threshold to nylon hairs, and the patient made many errors in twopoint discrimination on the fingers. Vibration sensation was present but joint sensation was mildly defective in the fingers.

Immediately before a tourniquet test on the right hand on February 15 the patient was unable to feel either $0.3 \mathrm{~g}$. or $0.5 \mathrm{~g}$. nylon hairs on the finger-tips, but made no errors with a $1 \mathrm{~g}$. hair. There were errors in discrimination of compass points on the fingers at $2 \mathrm{~cm}$. separation, but not at $2.5 \mathrm{~cm}$. When the fingers were firmly rubbed the patient described them as "a bit numb" but " not completely dead".

A sphygmomanometer armlet was then inflated to $220 \mathrm{~mm}$. $\mathrm{Hg}$ round the right arm above the elbow and resulted in rapid deterioration in sensation (Fig. 1C). Ischaemic paraesthesiae were absent altogether, and after five minutes touches with a $1 \mathrm{~g}$. hair could no longer be felt. At this time the patient first remarked that the sensation aroused by stroking or rubbing the finger-tips was changed, and that they felt more numb and dead than before the ischaemia.

Comment.-This was an advanced case of disseminated sclerosis with moderately severe symmetrical sensory loss in the hands. A pneumatic tourniquet above the elbow resulted in the development of complete anaesthesia to a $1 \mathrm{~g}$. hair after five minutes of ischaemia, with a corresponding increase in the subjective numbness of the skin, a more rapid change in sensation than had occurred in either of the previous cases.

"Time to Numbness" as an Index of Sensory Recovery.-From the results already described, it seemed possible that either improvement or deterioration in a patient's condition might be accompanied by changes in the "time to numbness", which could be recorded by repeated tests with a cuff. To investigate this further, two patients with cervical cord lesions were examined at regular intervals, and changes in the "time to numbness" compared with the results of formal sensory testing.

Case 4.-Fig. 2 shows the results of a series of tests in Case 4, a patient who was being treated by continuous head traction. The patient (N.H. No. 38405) had been admitted under the care of Dr. Denis Brinton with spastic weakness of all four limbs and mild symmetrical sensory loss in the hands. He was thought at first to 
Fig. 2 (Case 4).-Cervical cord tumour. Pneumatic tourniquet tests during pre-operative head traction showing " time to numbness" for right thumb (R.F.1) and fifth finger (R.F.5).

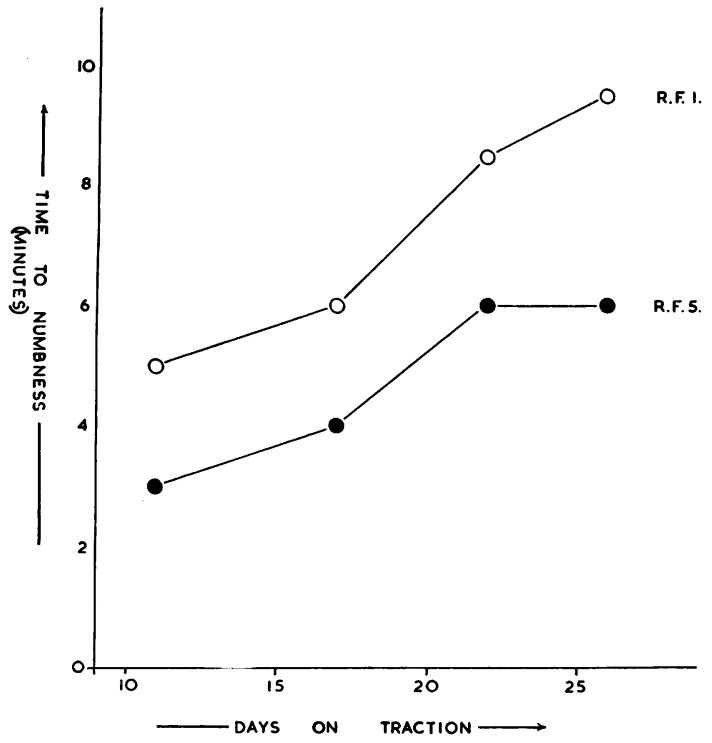

be suffering from cervical spondylosis, but at operation (Mr. Harvey Jackson) he was later found to have a small intramedullary endothelioma at the level of C4.

A tourniquet test was first carried out on July 23, 1953, after the patient had been on continuous traction for 11 days. At that time sensation was still impaired in both hands, particularly on the ulnar sides. The skin of the ring and fifth fingers felt " slightly numb " when it was touched, but on the thumb and on the index and middle fingers, touches were described as normal. Pain and temperature sensation were not obviously impaired in the hands, but the patient was unable to feel a $0.5 \mathrm{~g}$. nylon hair on any finger, and made errors in the discrimination of compass points $1 \mathrm{~cm}$. apart on the index. The vibration of a tuning fork was subjectively reduced in the fifth finger compared with the index and thumb. Joint sensation was defective in the fifth finger, but normal in the other digits.

When a sphygmomanometer cuff was inflated to $220 \mathrm{~mm}$. $\mathrm{Hg}$ round the right arm above the elbow no ischaemic tingling was felt. After three minutes the patient described sensation in the skin of the fifth finger as " a little worse than before" when it was rubbed, the other fingers being unchanged. After four minutes all digits except the thumb felt " a bit dead" to touch, and at five minutes the thumb was also affected, all digits feeling " dead" and "wooden" when they were rubbed together.

Six days later the tourniquet test was repeated. The patient himself considered that the right hand had improved and that the numbness of the ring and little fingers was less, but examination with nylon hairs and compass points revealed no change. When the pneumatic tourniquet was applied above the elbow, deterioration in sensation in the fifth finger was noticed after four minutes, numbness spreading rapidly across the hand to involve the thumb after six minutes. No ischaemic tingling was felt in this or in subsequent tests.

Five days later the patient was re-examined. Subjectively sensation in the right hand was unchanged, and there were still errors in discrimination or compass points at $1 \mathrm{~cm}$. separation on the index finger. A $0.5 \mathrm{~g}$. nylon thread was now occasionally perceived on the radial fingers, but replies were inconstant and the patient tired rapidly. The ischaemic test showed a definite change in that subjective numbness appeared later and spread more slowly across the hand. No change was noticed in the fifth finger until six minutes, and in the thumb the sensation aroused by touch was described as normal until eight and a half minutes.

A final test was carried out on the right hand four days later. The patient himself was not convinced that there had been any further improvement in sensation, and formal sensory testing revealed no change. After six minutes of ischaemia the fifth finger felt "very dead ", but the patient noticed no deterioration in the thumb until 10 minutes. After 11 minutes the thumb was still much less numb than the other digits.

Comment.-In a patient with a cervical cord lesion there was slight but definite improvement in sensation during head traction, which was shown more clearly by the use of ischaemia than by other sensory tests on the hand. Initially there was a difference in sensation between the two sides of the hand, and during traction improvement was more marked on the radial than the ulnar side. Both of these findings were subsequently explained by the discovery of a spinal cord tumour at the level of $\mathrm{C} 4$.

Case 5.-A series of ischaemic tests on Case 5 is shown in Fig. 3. The patient (N.H. No. 43140) had been admitted under the care of Dr. Purdon Martin with spastic weakness of all four limbs due to a large neurofibroma at the level of C2. Initially all forms of sensation were severely impaired in the limbs, and superficial sensory loss extended as high as the third

FIG. 3 (Case 5).-Cervical cord tumour. Post-operative tourniquet tests showing recovery of sensation in right hand.

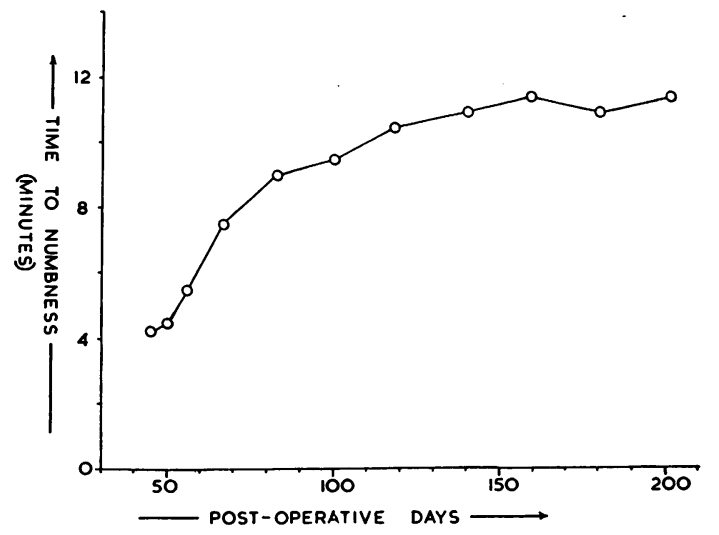


cervical dermatome, but after removal of the tumour by $\mathrm{Mr}$. Harvey Jackson sensation rapidly improved.

The first tourniquet test was carried out on the right hand on April 13, 1954, 45 days after operation. At this time the fingers did not feel abnormal when they were rubbed, and the touch of a $0.3 \mathrm{~g}$. hair was perceived on the finger-tips. Painful and thermal stimuli were also appreciated normally. The patient still made errors in two-point discrimination at $0.7 \mathrm{~cm}$. separation on the finger-pads, but not at $1 \mathrm{~cm}$. Vibration and joint sensation were normal. When a sphygmomanometer cuff was inflated to $210 \mathrm{~mm}$. $\mathrm{Hg}$ round the arm above the elbow, no ischaemic tingling was felt. Four minutes after the onset of ischaemia the patient had noticed no change in sensation, but a few seconds later the fingertips began to feel numb when they were rubbed together, and by five minutes they were described as "dead fingers".

On the 50th post-operative day a second tourniquet test gave a similar result, but on the 56th day the " time to numbness" had increased to five and a half minutes, and on the 67 th day to seven and a half minutes. By this time the patient was usually correct in two-point discrimination at $0.7 \mathrm{~cm}$. separation although he tired easily. When tested with nylon threads, the patient's performance was also greatly influenced by fatigue, and changes during ischaemia were much less constant and reproduceable than the onset of subjective numbness. For this reason the use of nylon threads during ischaemia was abandoned in later tests.

By the 100th post-operative day the patient made only occasional errors in discrimination of compass points at $0.5 \mathrm{~cm}$. separation on the fingers, and the " time to numbness" had increased to 10 minutes. On this occasion the patient first noticed ischaemic paraesthesiae, and described faint tingling beginning two minutes after the onset of ischaemia and lasting for four minutes. Ischaemic tingling recurred in all tests after this time.

Between the 100th and 200th post-operative days there was little change in sensation in the hand. The patient continued to make occasional errors in twopoint discrimination when fatigued, and the "time to numbness" did not increase beyond 12 minutes.

Comment.-After the removal of a high cervical cord tumour, the patient was examined at regular intervals for six months. At the end of this time it seemed that improvement in sensation had ceased although recovery was stillincomplete and the patient continued to make errors in two-point discrimination when fatigued. During the period of observation there was a progressive increase in the "time to numbness" which appeared to reflect the gradual improvement in function of the cervical cord.

\section{Patients with Cerebral Lesions}

Six patients with cerebral lesions were examined. Four of them were suffering from cerebral vascular disease ; the two remaining patients were examined shortly after the successful removal of cerebral tumours.

Shortening of the "Time to Numbness".- In three patients the " time to numbness" was reduced in the affected hand, but in two of them the reduction was small and required simultaneous ischaemia of both arms to demonstrate the abnormality satisfactorily.

Case 6.-Mr. K. I., aged 24 (N.H. No. 50386), a right-handed police officer, was admitted to the National Hospital under the care of Dr. Denis Williams on February 4, 1954, with a history of weakness of the right arm and leg of sudden onset with loss of speech in August, 1952. Some recovery of function had followed, but six months after the onset the patient began to suffer from right-sided and generalized fits.

On admission in February, 1954, the patient showed no evidence of intellectual deterioration, but mild expressive dysphasia was present, with a moderately severe right hemiparesis affecting the arm more than the leg. Sensory loss was restricted to the right side of the face and to the right hand. The patient's blood pressure was $105 / 60 \mathrm{~mm}$. $\mathrm{Hg}$, and lumbar puncture, air encephalography, and carotid arteriography failed to reveal any abnormality. An electroencephalogram was considered to support the clinical diagnosis of a cerebral vascular lesion.

In spite of minimal dysphasia the patient proved to be an intelligent and reliable witness and detailed sensory examination before ischaemia on February 23 gave the following results. Subjectively the skin of the right hand felt slightly different to the left, but the patient denied that it was actually numb to touch. Light touches with 0.3 and $0.5 \mathrm{~g}$. nylon hairs were frequently missed on the right fingers, but there were no errors with a $1 \mathrm{~g}$. hair. Pinpricks were appreciated everywhere on the right arm and hand, but on the fingers the sensation was subjectively reduced compared with the left. Discrimination of compass points was severely defective on the right fingers and errors were frequent at $2 \mathrm{~cm}$. separation. Passive movements of the right digits were perceived, but the patient's answers were slower and more hesitant than with the left fingers ; the patient was also slow in identifying coins placed in the right hand.

A pneumatic tourniquet was inflated to $220 \mathrm{~mm}$. $\mathrm{Hg}$ round the right arm above the elbow and within five minutes the patient began to miss touches with a $1 \mathrm{~g}$. hair on the index finger. After seven and a half minutes he reported that the feeling was "definitely less" when the fingers were rubbed; by nine minutes the skin felt "very dead" and the $1 \mathrm{~g}$. hair was only occasionally perceived. No ischaemic tingling was felt at any stage.

Comment.-In this patient there was uncertainty as to the pathological nature of the lesion; the sudden onset of symptoms and subsequent epilepsy had suggested the possibility of spontaneous intracerebral haemorrhage, but no vascular anomaly was demonstrated by arteriography. At the time of examination, 
sensory loss in the right hand was mild, but it became appreciably worse within five to seven minutes of the inflation of a pneumatic tourniquet round the arm.

Case 7.-Mr. C. W. (N.H. No. 44726), a right-handed man aged 54, was admitted to the National Hospital under the care of Dr. Purdon Martin on May 4, 1953, with a history of sudden complete paralysis of the right arm and leg with loss of speech in 1948 . Recovery of function had been slow and incomplete, and on admission in May, 1953, moderately severe residual dysphasia was present with spastic weakness of the right limbs and considerable sensory disturbance affecting both the arm and leg. The patient's blood pressure was $130 / 80$ $\mathrm{mm}$. $\mathrm{Hg}$ and the fundi showed severe retinal arteriosclerosis. Neither lumbar puncture nor carotid arteriography revealed any abnormality, and an electroencephalogram was considered to support the clinical diagnosis of cerebral thrombosis.

Sensory testing was difficult owing to the patient's dysphasia, but examination before ischaemia on May 11 gave the following results. There was subjective numbness of the skin of the right hand and forearm, and pinpricks were reported as dull below the wrist. Fine nylon hairs were not felt on the right fingers and replies were variable with heavier stimuli. Two-point discrimination and joint sensation were severely defective in the right fingers and coins placed in the right hand could not be distinguished. No abnormality was detected in the left hand.

Two sphygmomanometer cuffs were inflated simultaneously round the right and left arms so that the effects of ischaemia could be compared on the two sides. Ischaemic tingling was not significantly different in the two hands, but eight minutes after the onset of ischaemia the patient noticed a change in the right fingers and described them as very dead when they were touched. The left fingers were not subjectively numb to a firm touch until 11 minutes, at which time the patient described the difference between the two hands as much greater than before the experiment. Testing with nylon hairs was not attempted during ischaemia owing to the variability of the patient's replies beforehand.

Comment.-In a patient with moderately severe right-sided sensory loss due to cerebral thrombosis, simultaneous ischaemia of the normal and affected arms increased the subjective difference in sensation between the two sides, a change being apparent to the patient after eight minutes.

Case 8.-Mr. J. G. (N.H. No. 45599), a right-handed man aged 49, was admitted to the National Hospital under the care of Dr. Purdon Martin on June 23, 1953, with the history that five weeks before admission he had suddenly felt giddy and within an hour developed mild right-sided weakness and disturbance of speech without loss of consciousness. At the time of his admission the patient was already greatly improved and speech was normal, but slight spastic weakness of the right arm and leg remained. Sensation was subjectively impaired in the skin of the right hand and foot although tests with $\bar{Z}$ compass points and fine nylon hairs revealed no definite $\stackrel{\mathbb{D}}{\leftarrow}$ sensory loss. The blood pressure was $190 / 110 \mathrm{~mm}$. Hg and the fundi showed moderately severe retinal arterio- of sclerosis. Other investigations supported the clinical $\overline{\bar{c}}$ diagnosis of cerebral thrombosis.

Examination before ischaemia on July 6 showed the sensory disturbance in the right hand to be almost $\stackrel{\rho}{\supset}$ entirely resolved, and the patient himself was no longer aware of any abnormality. There were no differences $\underset{\gtrless}{\gtrless}$ between the right and left hands for pinpricks, thermal stimuli or light touches, and no sensory inattention to simultaneous stimuli could be demonstrated. Compass points were correctly distinguished at $0.5 \mathrm{~cm}$. separation on the fingers, and joint sensation was normal. Coins $\overline{\bar{\sigma}}$ were recognized without difficulty when placed in either $\vec{D}$ hand, but the vibration of a tuning fork was felt less strongly on the right fingers than the left.

Two pneumatic cuffs were inflated simultaneously $\vec{\circ}$ round the right and left arms above the elbows. A pressure of $230 \mathrm{~mm}$. $\mathrm{Hg}$ proved adequate for arterial arrest $\vec{\omega}$ in spite of the patient's hypertension. Ischaemic tingling was much reduced and delayed on the right, but no other sensory disturbance was noted until $12 \frac{1}{2}$ minutes when $\frac{7}{0}$ sensation in the right fingers became "a bit dull " to $\vec{\infty}$ touch. At 14 minutes the patient remarked on "a bi difference" between the two hands and described the right as having "wooden fingers". By $15 \frac{1}{2}$ minute $\$$ ㅇ when subjective numbness first appeared in the finge tips on the left, the whole right hand felt dead ang some numbness of the skin had begun to spread abote the right wrist.

Comment.-When this patient was examined sever weeks atter a cerebral thrombosis, sensation in the affected hand seemed normal save that a tuning fork was felt less strongly than on the other hand. When ischaemia was induced simultaneously in both arms, sensory loss occurred first in the finger-tips on the affected side and spread so rapidly that the whole

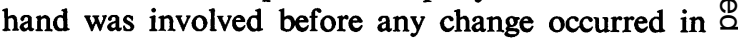
the normal fingers. Ischaemic paraesthesiae were also reduced on the affected side.

Lengthening of the "Time to Numbness".-From the results described above it seemed possible that the "time to numbness" would be reduced in any patient with pre-existing sensory loss, regardless of ? the level of the lesion, but in two of the patients with cerebral lesions the expected reduction was not found.

Case 9.-Mr. C. R. (N.H. No. 46951), a right-handed 0 man aged 51, was admitted to the National Hospital under the care of Sir Charles Symonds on August 14, $\frac{D}{O}$ 1953, with a short history of attacks of unconsciousness and of increasing weakness of the right arm and leg. A N large left parietal meningioma was successfully removed by Mr. McKissock on August 26, but after operation $\mathrm{N}$ considerable weakness of the right limbs remained, $N$ with moderately severe residual sensory disturbance. 
When an ischaemic test was carried out on the 35th post-operative day, no dysphasia remained and the patient was able to cooperate well in the sensory examination. Before ischaemia there was no subjective numbness of the right hand, although light touches were frequently missed and pinpricks did not feel as sharp as on the left. There were many errors in the discrimination of compass points at $0.7 \mathrm{~cm}$. separation on the right fingers. Joint sensation was defective in the fingers and the patient was unable to identify coins placed in the right hand. The inflation of pneumatic cuffs round the arms above the elbows resulted in subjective numbness of the right fingers after $15 \frac{1}{2}$ minutes compared with $16 \frac{1}{2}$ minutes on the left. Ischaemic tingling was present but reduced on the right compared with the left.

Case 10.-Mr. R. H. (N.H. No. 46118), a left-handed man aged 37, was admitted to the National Hospital under the care of Dr. Purdon Martin on July 20, 1953, six months after a right hemiplegia of sudden onset without loss of consciousness. Recovery had been rapid, and on admission only trivial right-sided weakness remained. The blood Wassermann reaction was strongly positive, but the cerebrospinal fluid was normal.

Before an ischaemic test on July 27 appreciation of fine nylon hairs was normal on the right fingers and no sensory inattention was present. Two-point discrimination and joint sensation were also normal, but the patient had great difficulty in identifying coins placed in the right hand, whereas he made no errors with the left hand. Pain and temperature sensation was normal. Pneumatic cuffs were inflated simultaneously round both arms and resulted in ischaemic paraesthesiae which were equal in intensity on the two sides. After 10 minutes subjective numbness was present in the finger-iips on the left at which time the right fingers still felt normal when they were touched or rubbed. No change was recognized in the right fingers until 12 minutes, but after this time subjective numbness to touch was present and was considered to be equal on the two sides.

Comment.-In the first of these two patients considerable sensory disturbance was present in the affected hand without a significant decrease in the "time to numbness". In the second patient the only sensory function affected was a discriminative one, astereognosis being present without any rise in tactile threshold. In this patient the "time to numbness" was slightly longer in the affected hand than in the normal one.

"Time to Numbness" during Sensory Recovery.The apparent contradiction between the last two patients and those examined previously was resolved when Case 11 was studied.

Case 11.-The patient (N.H. No. 46232), a righthanded woman aged 45 , who was under the care of Sir Charles Symonds, underwent craniotomy for a right parietal meningioma on July 30, 1953; the tumour was successfully removed by Mr. McKissock, but severe left-sided sensory disturbance persisted. Sensation in the left hand was examined at regular intervals for six months, at the end of which time recovery was still incomplete. The results of ischaemic tests during this period are shown in Fig. $4 ; 10$ control observations on the right hand were made, but these were carried out separately from those on the left and no simultaneous bilateral tests were used.

On the 46th post-operative day, when the first tourniquet test was carried out, the patient's speech and mental state were normal, and she was able to cooperate well in the examination. A partial left homonymous hemianopia was present, with a tendency to neglect objects to the left. Moderately severe left-sided weakness was present affecting the face, arm, and leg, but there was no denial of disability or gross neglect of the left limbs. In the left arm and hand there was no loss of appreciation of pinpricks, but left-sided inattention was present on bilateral stimulation. The left fingers felt numb and tingled when they were firmly touched, but the palm and dorsum of the hand were subjectively normal. Fine nylon hairs were not felt on the left fingers, and tactile localization, two-point discrimination, and joint sensation were severely impaired. When a sphygmomanometer cuff was inflated to $210 \mathrm{~mm}$. $\mathrm{Hg}$ round the left arm above the elbow, some ischaemic tingling resulted, but this was short-lived, and after four minutes the patient described the sensation produced by rubbing the finger-tips as "much less" than before the test.

On the 53rd post-operative day there had been some improvement in sensation and the patient was able to localize touches to the correct finger although the skin still felt numb and tingled to touch. When a pneumatic tourniquet was inflated round the arm the finger-tips again felt "dead" to touch after four minutes of ischaemia.

By the 60th day the sensation aroused by rubbing the thumb seemed normal although this procedure still caused tingling in the other digits. However, after five and a half minutes of ischaemia all the digits felt numb to touch at their tips, and no difference could be detected between the thumb and fingers.

By the 81st day the "time to numbness" on the left had increased to seven and a half minutes and by the 110th day to 11 minutes. Although this figure now approximated to the "time to numbness" of the right hand, sensation on the left was still obviously impaired. Subjectively the left fingers felt normal, but a 1 g. nylon hair was only occasionally perceived, and pinpricks felt less sharp than on the right. Sensory inattention to bilateral stimuli was still present and two-point discrimination and joint sensation were grossly defective.

As shown in Fig. 4, subsequent tourniquet tests gave figures for the " time to numbness" in the affected hand which were consistently higher than in the control hand. In the final test on the 166th day ischaemia produced no change in the sensation of touch on the left fingers until 18 minutes, as compared with 13 minutes for the right fingers. In spite of this, sensation in the left hand was still abnormal. There was no subjective numbness 
Fic. 4 (Case 11).-Right parietal meningioma. Post-operative tourniquet tests showing recovery of sensation in left hand and control

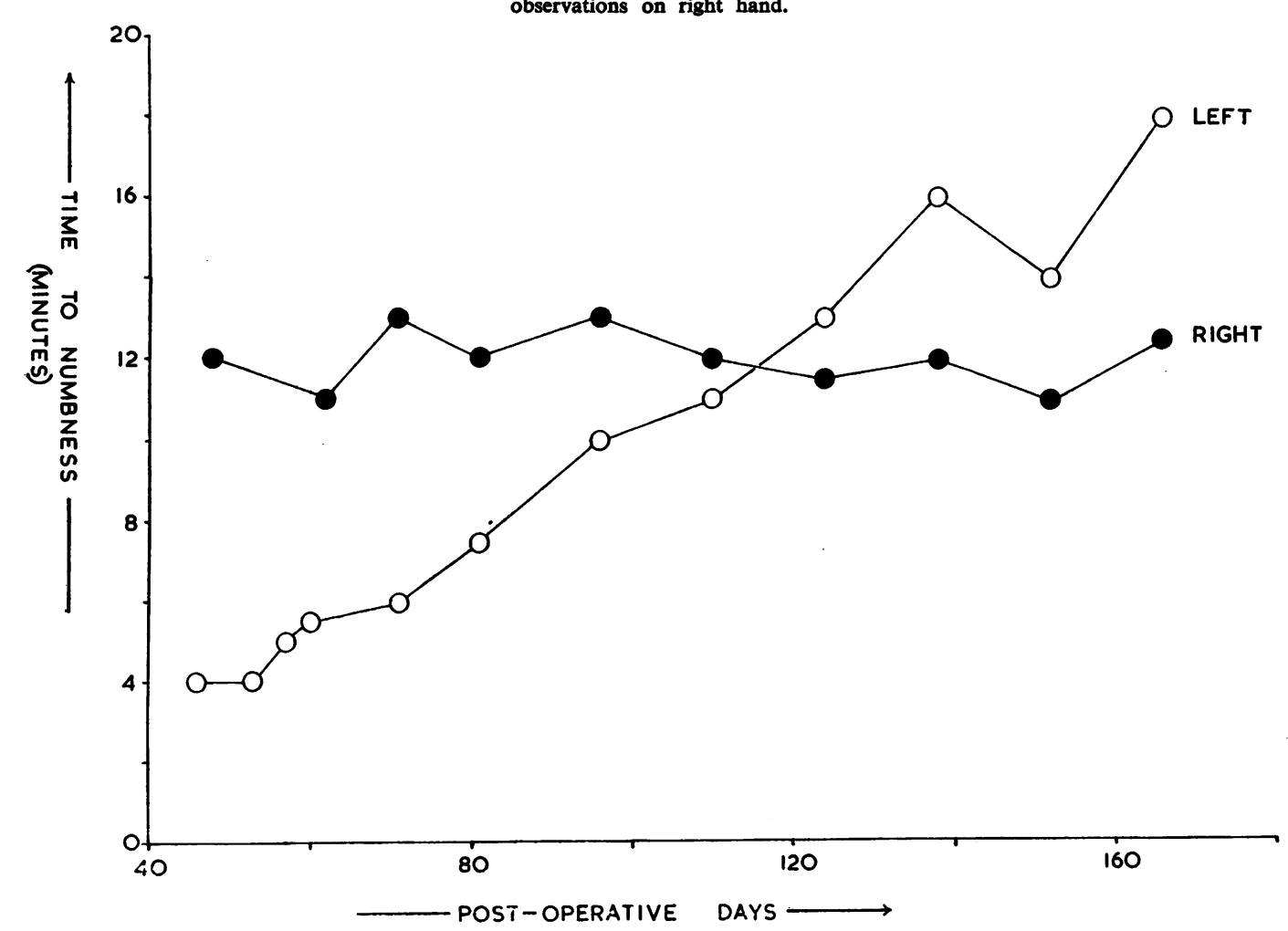

of the skin and no sensory inattention, but the $0.3 \mathrm{~g}$. hair.was rarely perceived and there were occasional failures with the $0.5 \mathrm{~g}$. hair. The patient still made errors in discrimination of compass points at $0.5 \mathrm{~cm}$. separation but not at $0.7 \mathrm{~cm}$. Joint sensation in the left fingers was defective and the patient was unable to distinguish the texture of different materials when she handled them with her left hand, although she accomplished this without difficulty with her right hand.

Comment.-After the removal of a right parietal tumour sensation in the left hand was slow to recover. At first the "time to numbness" in the left hand was shorter than normal, but as sensation improved the time increased, until after four months it consistently exceeded the normal. At this stage the tactile threshold was only slightly raised, but discriminative sensation was severely affected. Since the patient was unable to distinguish the texture of objects held in the hand, a similar difficulty must have existed when she attempted to assess the sensation aroused by rubbing the finger-pads during ischaemia. It is, therefore, possible that the first changes in sensation produced by ischaemia were ignored on the affected side, resulting in an apparent increase in the "time to numbness".

\section{Discussion}

In a previous paper it was shown that in patients with peripheral nerve and dorsal root lesions affecting the hand, the inflation of a pneumatic tourniquet round the arm produced abnormally rapid deterioration of sensation in the distribution of the affected nerve or root. This was considered to be due to the combined effect of the two partial nerve blocks at different levels, one being at the level of the lesion and the other in the peripheral segment of nerve artificially deprived of its blood supply. The present work supports this view, and shows that in patients with spinal or cerebral lesions a peripheral nerve block may also add its effect to that of the central lesion, and result in sensory loss in excess of that caused by either one alone.

In a normal subject, ischaemia of the arm must be maintained for 10 or 15 minutes to cause sensory loss, whereas in a patient with normal peripheral nerves but with a cervical cord lesion, sensory deterioration occurs much earlier during ischaemia. This implies that there is in the normal sensory system a considerable reserve of function, and that a substantial-proportion of the afferent fibres from 
a given area must fail to conduct impulses before sensory loss can be expected.

From this conclusion several deductions of clinical importance emerge. In the first place it is clear that small nervous lesions are unlikely to give rise to detectable sensory loss ; this must explain many clinical instances of mild peripheral nerve lesions without changes in sensation. Absence of sensory loss in dorsal root lesions is also easily explained since there is, in any case, considerable spatial overlap between adjacent roots. If survival of one half or two-thirds of the normal number of nerve fibres from a given area is sufficient to provide apparently "normal" sensation, it is hardly surprising that complete loss of a single dorsal root does not necessarily result in sensory loss.

In clinical neurology one might also expect to find examples of sensory loss due to the combined effect of two lesions at different levels. It is not uncommon for a patient with disseminated sclerosis to notice that a foot "goes to sleep " with abnormal rapidity when the legs are crossed, a phenomenon which is directly comparable with the effect of a pneumatic cuff, and it is likely that there are other conditions in which the existence of two lesions may result in unusual patterns of sensory loss. For example, the occasional occurrence of sensory loss of a peripheral nerve or dorsal root distribution in a patient with a cerebral tumour may be due to the superimposition of the effects of two lesions at different levels. Certainly a very mild disturbance at a peripheral level would be sufficient to produce its effect in the presence of diffuse sensory loss due to a cerebral lesion. The occasional finding of a sensory level in the dorsal region in a patient with cervical spondylosis might be explained in the same way, a mild and unsuspected dorsal disc protrusion being sufficient to cause this false localizing sign in the presence of some pre-existing sensory disturbance due to a lesion at a higher level.

In the previous paper it was suggested that the use of a pneumatic tourniquet might prove to be of practical value in the investigation of disturbances of sensation. In the diagnosis of peripheral nerve lesions, the test was particularly helpful in patients whose sensory loss was initially too slight and illdefined to allow accurate demarcation of the area of skin involved. In such cases the effect of ischaemia on adjacent areas of normal skin provided the necessary control against which the behaviour of damaged nerve could be assessed. However, the patients with spinal and cerebral lesions described in the present paper have mostly shown diffuse sensory loss throughout the hand, and in them the effects of ischaemia cannot be interpreted so easily.
Since the susceptibility of peripheral nerve to ischaemia varies widely in normal subjects, a single observation of the " time to numbness" may not be helpful unless the patient's normal susceptibility to ischaemia is known.

Although the time of onset of ischaemic sensory loss varies considerably in different individuals, this is not the case when repeated tests are carried out on the same subject. For example, Fig. 4 shows that in Case 11 the "time to numbness" in the patient's unaffected hand was never less than 11 minutes and never more than 13 minutes in 10 consecutive tests over a period of four months. This may be contrasted with the normal range of eight to 18 minutes found in 50 different subjects (Gilliatt and Wilson, 1954). Thus repeated tourniquet tests may be of considerable value in recording slow changes in sensation over long periods of time, and in fact the method has proved sufficiently accurate to reveal gradual changes in the condition of a spinal cord lesion, even when these have been slight and difficult to demonstrate by formal sensory testing. In the case of cerebral lesions, however, a complicating factor has been introduced by the nature of the ischaemic test. The appreciation of a changed sensation in the skin when it is touched or rubbed is a discriminative function of the cerebral cortex, and it is, therefore, defective in patients whose cerebral lesions render them incapable of discriminating texture or shape. When such patients have been tested with ischaemia, the onset of numbness in the fingers has been acknowledged only after a considerable delay and the "time to numbness" has appeared to be longer than normal.

Except in this last group of patients, the onset of subjective numbness has usually proved easier to measure than changes in the tactile threshold. In the present series of tests, as in previous work, paraesthesiae have been found to exert a profound effect on the perception of fine nylon hairs, and tingling which has barely been perceptible to the patient has sometimes caused a sharp rise in tactile threshold. For this reason patients with spontaneous tingling may appear to improve during ischaemia if this causes paraesthesiae to subside ; alternatively patients in whom a cuff produces intense paraesthesiae may fail altogether to appreciate light touches after one or two minutes of ischaemia, but recover later as paraesthesiae decline. On the other hand the onset of subjective numbness of the skin has proved easily recognizable even in the presence of spontaneous tingling, and throughout the present work great reliance has been placed on the patient's description of the sensation aroused by a firm touch or by rubbing the skin. 
Sinclair and Hinshaw (1951) reported that ischaemic tingling was diminished or absent in patients with spinal or cerebral lesions causing defective tactile sensibility, and suggested that "It may even be that a diminution in the sensation of tingling affords a more sensitive test for such defects than standard testing methods". However, the present work suggests that the relation between tingling and tactile loss is not a simple one. In the group of spinal cord lesions, ischaemic tingling was short-lived or absent in all the patients with a raised tactile threshold and a reduced "time to numbness" (Cases 2, 3, and 4), and in Case 5 ischaemic tingling returned as sensation improved after operation. In those with cerebral lesions, however, ischaemic tingling was absent in only one case (Case 6), and reduced or delayed in two (Cases 8 and 9). In Cases 7 and 11 ischaemic tingling was present although there was considerable sensory loss and a reduced " time to numbness" in each case.

Marshall (1952) found that in patients with sciatica due to lumbar disc degeneration, ischaemic tingling sometimes occurred earlier in the affected foot than in the normal one. Marshall explained his results by suggesting that there were degenerative changes in the distal segments of the affected nerve fibres which rendered them more irritable during ischaemia. His results could, however, be explained by the simple additive effect of two separate irritative foci along the course of the nerve, one at the level of the lesion and the other under the pneumatic cuff.

If, in patients with spinal and cerebral lesions, irritable foci at different levels can exert an additive effect, the intensity of ischaemic paraesthesiae would presumably depend upon the response of ischaemic nerve peripherally and upon the amount of irritation and spontaneous discharge occurring in the central lesion. The latter would not necessarily be related to the severity of the lesion in terms of other sensory defects.

\section{SUMMARY}

In 11 patients with sensory loss in the hand due to spinal or cerebral lesions, the circulation to the affected arm has been arrested by a pneumatic tourniquet.

When a partial peripheral nerve block has been produced in this way, its effect on sensation has been added to that of the central lesion and has resulted in sensory loss in excess of that produced by either one alone.

In patients with spinal cord lesions, repeated tourniquet tests provide a sensitive method of recording improvement or deterioration in the condition of the patient. In those with cerebral lesions there are, however, objections to this form of test.

I am indebted to members of the staff of the Nationalo Hospital for permission to publish details of cases under their care. I also wish to thank Dr. E. A. Carmichaelत̄ for helpful criticism and advice.

\section{REFERENCES}

Gilliatt, R. W., and Wilson, T. G. (1954). Journal of Neurology, Neurosurgery and Psychiatrv, 17, 104.

Marshall, J. (1952). Ibid., 15, 242.

McAlpine, D. (1954). Arch. Middx. Hosp., 4, 1.

Sinclair, D. C., and Hinshaw, J. R. (1951). Quart. J. exp. Psychol., 3,49.

Weddell, G., and Sinclair, D. C. (1947). Journal of Neurology. Neurosurgery and Psychiatry, 10, 26.

\section{THE FEBRUARY (1955) ISSUE}

The February (1955) issue contains the following papers :-

Variations in the Basophilia of Nerve Cells Associated with Increased Cell Activity and Functional Stress. Lárus Einarson and Erik Krogh.

Argyrophile Bodies in the Human Spinal Cord. Marion C. Smith.

Morphological Nervous Changes in Survivors of Severe Jaundice of the Newborn. L. Crome.

Unusually Severe Lesions in the Brain Following Status Epilepticus. A Meyer, E. Beck, and M. Shepherd.

Prefrontal Leucotomy and the Anticipation of Pain. Alick Elithorn, Mialcolm F. Piercy, and Margaret A. Crosskey. Intellectual Changes Following Temporal Lobectomy for Psychomotor Epilepsy. Victor Meyer and Aubrey J. Yates. Visual Hallucinations of the Self in Organic Disease. Kenneth Dewhurst and John Pearson.

Basilar Impression. D. G. Phillips.

Some Observations on the Electroencephalogram in Cerebral Tumours. J. H. D. Millar.

Alzheimer's Disease with Acne Rosacea in One of Identical Twins. Esther A. Davidson and E. Elizabeth Robertson.

A number of copies are still available and may be obtained from the Publishing Manager, British Medical Association, Tavistock Square, W.C.1, price 12s. 6d. 